\title{
DIE GESELLSCHAFTLICHE INWERTSETZUNG VON KULTURERBE
}

\author{
Parallelen zwischen Denkmalschutz und Naturschutz
}

\begin{abstract}
Nicht erst im Europäischen Jahr für Kulturerbe (2018) werden historische Landschaftsgärten und andere Kulturdenkmäler weniger als Kostenfaktor gesehen, sondern als Ressource, die als weicher Standortfaktor zu Wettbewerbsfähigkeit, Entstehung von Arbeitsplätzen und regionaler Entwicklung führt. Direkter ökonomischer Nutzen entsteht beispielsweise in den Bereichen Tourismus, Immobilienwirtschaft und Exportwirtschaft. Indirekter Nutzen kann sich durch Beiträge zum menschlichen Wohlbefinden, zum Heimatgefühl oder zur historischen Identität einer Region entfalten. Entsprechend wird beispielsweise von der Horizon 2020 Expert Group on Cultural Heritage der Europäischen Kommission gefordert, Kulturerbe als Querschnittsthema in verschiedenen Politikfeldern zu verankern. Die Debatte um die gesellschaftliche Aufwertung des Kulturerbes weist erhebliche Parallelen zur ökonomischen Aufwertung von Ökosystemen auf, die den Naturschutz im Zuge des globalen Umweltwandels stark beeinflusst hat. Mit der Erfindung des Konzepts der »Ökosystemleistungen « wurde ab der Jahrtausendwende eine ökonomische Argumentation zur Erhaltung der Natur eingeführt, die auf die globale Umwelt- und Entwicklungspolitik starken Einfluss ausgeübt hat und die in diesem Beitrag beleuchtet werden soll. Im Vergleich der Entwicklungen im Denkmalschutz und Naturschutz leitet der Beitrag Aussagen zu den Chancen und Risiken sowie Entwicklungspfade für eine gesellschaftliche Inwertsetzung von Kulturerbe ab.
\end{abstract}

Even before the European Year of Cultural Heritage (2018) historic landscape gardens and other cultural monuments were viewed less as a cost factor and more as a resource that leads as a soft location factor to competitiveness, the creation of jobs and regional development. A direct economic benefit is generated, for instance, in the areas of tourism, the real estate sector and the export industry. An indirect benefit can emerge through contribu-

Im Text werden Maskulinum und Femininum verwendet, wenn es um Personen geht. Gemeint sind grundsätzlich alle Menschen, gleich welcher Geschlechtsidentität sie sich zugehörig fühlen. 
tions to human well-being, a sense of place or the historic identity of a region. Consequently, the Horizon 2020 Expert Group on Cultural Heritage of the European Commission has called for the embedding of cultural heritage as an overarching topic in various policy areas. The debate about the social valorisation of cultural heritage presents numerous parallels to the economic valorisation of ecosystems that has very much influenced nature conservation in the course of global environmental change. With the introduction of the concept of »ecosystem services « economic arguments in favour of nature conservation have been put forward from the turn of the millennium. They have had a major impact on global environmental and development policies. They are examined in this article. In its comparison of the developments in the protection of listed buildings and monuments and nature conservation, the article derives statements on the opportunities and risks along with development pathways for the social valorisation of cultural heritage.

\section{Einleitung}

In der englischen Sprache beziehen sich die Begriffe "Heritage« und »Conservation « auf natürliche und kulturelle Aspekte gleichermaßen. Dies weist darauf hin, dass Denkmalpflege und Naturschutz auf vielfältige Weise miteinander verbunden, wenn nicht sogar untrennbar sind, da beiden die Bewahrung des Kultur- bzw. Naturerbes ein Anliegen ist (Plachter/Rössler 1995; Lowenthal 2005). Beide sind häufig Konflikten durch vielfältige Nutzungsansprüche ausgesetzt (etwa von Seiten der Landwirtschaft oder der Stadtentwicklung), beide erfordern ein aktives Management und werden durch gesetzliche Vorschriften, öffentliche und private Fördermittel sowie durch ehrenamtliches Engagement vorangetrieben. Zunehmend werden Denkmalpflege und Naturschutz als Einheit gesehen, beispielsweise in der UNESCO-Welterbekonvention, die sowohl Kultur- als auch Naturdenkmäler einbezieht und historische Gärten, Agrarlandschaften oder spirituelle Orte als gemeinsame Kultur- und Naturdenkmäler unter Schutz stellt (Rössler 2006). Trotz dieser Gemeinsamkeiten sind jedoch viele der wissenschaftlichen, politischen und praktischen Diskurse zwischen Denkmalpflege und Naturschutz getrennt (Harrison 2015). Dies ist auf unterschiedliche Weltbilder im Hinblick auf Natur und Kultur, auf unterschiedliche Wertvorstellungen, aber auch auf unterschiedliche Vorstellungen über Schutzstrategien zurückzuführen (im Naturschutz wird eher der Aspekt der Dynamik bzw. Weiterentwicklung betont, in der Denkmalpflege eher der Aspekt der Bewahrung) (Lowenthal 2005).

Die ökonomische Inwertsetzung von Natur- und Kulturerbe ist ein Diskurs, der im Naturschutz seit ca. 20 Jahren geführt wird, in der Denkmalpflege hingegen aber erst seit Kurzem (siehe z.B. Throsby 2010). Dieser Beitrag reflektiert Erfahrungen aus dem EU-Projekt Sustaining Futures for Europe's Heritage in Cultural Landscapes (Plieninger et al. 2015) im Hinblick auf die künftige Rolle der Denkmalpflege. Er hat zum Ziel, anhand des Vergleichs mit dem Naturschutz Chancen, Risiken und Entwicklungspfade für eine gesellschaftliche Inwertsetzung von Kulturerbe zu beleuchten. Im nächsten Abschnitt beschreibe ich derzeit 


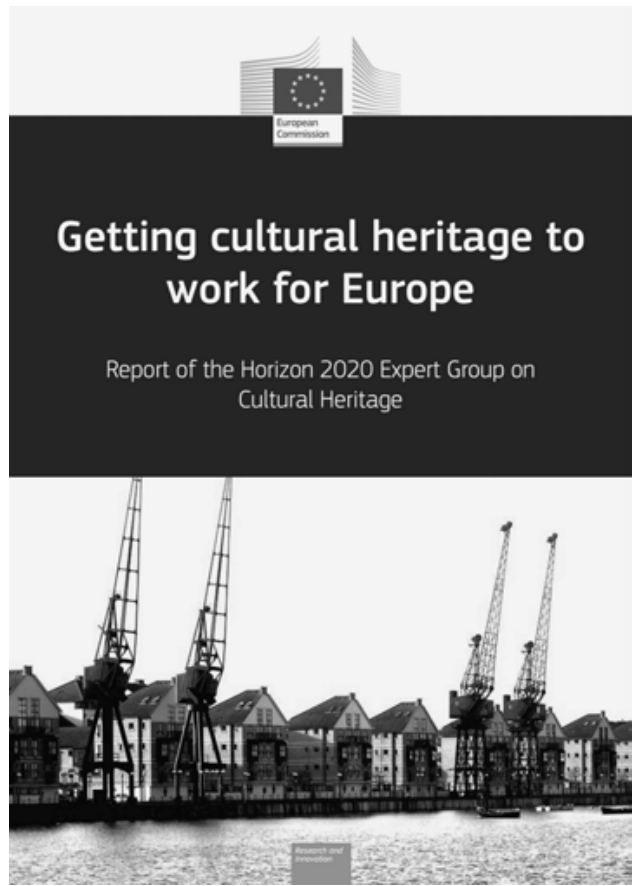

1 Der Bericht der EU-Expertengruppe zu Kulturerbe.

auf EU-Ebene laufende Bemühungen um eine gesellschaftliche Neuinterpretation der Denkmalpflege. Im Anschluss schildere ich ähnliche, aber zeitlich vorhergehende Diskussionen im Naturschutz, die sich um das Konzept der »Ökosystemleistungen« drehen. Schließlich gehe ich auf Chancen und Risiken einer Übertragung auf die Denkmalpflege ein.

\section{Gesellschaftliche Neuinterpretation von Denkmalpflege}

Im Jahr 2015 veröffentlichte das Direktorat Forschung und Innovation den Bericht Getting Cultural Heritage to Work for Europe (Europäische Kommission 2015, Abb.1), der die Arbeit einer Expertengruppe unter Leitung des früheren EU-Kommissars für Forschung, Wissenschaft und Technologie, Philippe Busquin, präsentierte. Auftrag der Arbeitsgruppe war es, dazu beizutragen, das Potential des Kulturerbes für Europa deutlich weiterzuentwickeln. Der Bericht war insbesondere dahingehend einflussreich, dass aus ihm finanziell sehr umfangreiche Forschungs- und Innovationsförderprogramme im Horizon-2020-Forschungsrahmenprogramm hervorgingen. Er hebt hervor, dass das Interesse an Denkmalpflege ständigen Schwankungen unterliegt, sich über die vergangenen 200 Jahre aber fest in Europa etabliert hat. Dabei richtet sich das gesellschaftliche Interesse nicht auf Denkmalobjekte und Museen allein, sondern auch auf immaterielle Kulturgüter sowie die Bewah- 
rung ganzer Ensembles oder Kulturlandschaften (Ahmad 2006). Zentrales Argument des Expertenberichts ist, dass das europäische Kulturerbe nicht nur um seiner selbst willen zu erhalten ist, sondern auch eine lebenswichtige Ressource und einen wichtigen Wettbewerbsvorteil gegenüber dem Rest der Welt darstellt. Damit soll die Denkmalpflege heute und in der Zukunft ein fester Bestandteil der zentralen Wirtschaftsstrategie der EU (»Europa 2020 «) für intelligentes, nachhaltiges und inklusives Wachstum werden (Europäische Kommission 2018).

Ein solches Verständnis von Kulturerbe, vor allem die Einbettung in die übergeordnete Wirtschaftsstrategie der EU, stellt eine Neuinterpretation der Rolle von Denkmalpflege dar: Lange wurde die Pflege von historischen Landschaftsgärten und anderen Denkmälern als Kostenfaktor betrachtet, der aus Gründen der Identitätsstiftung und zur historischen Erinnerung und Dokumentation zu finanzieren sei, dem aber kein messbarer ökonomischer Gegenwert gegenüberstehe (Lowenthal 2005). Nun hingegen interpretiert der Expertenbericht Kulturerbe als Beitrag zum Bruttosozialprodukt von Gesellschaften, zu Wachstum, zu Wettbewerbsfähigkeit und zum Wohlbefinden von Gesellschaften. Entsprechend wird argumentiert, dass Denkmalpflege in verschiedensten Politikfeldern Berücksichtigung finden und nicht mehr als Kostenfaktor, sondern als Produktionsfaktor definiert werden solle, als »strategische Ressource für ein nachhaltiges Europa« (Europäische Kommission 2015).

Gemäß dieser Interpretation entfaltet das Kulturerbe sowohl direkten als auch indirekten Nutzen. Zu den wichtigsten direkten Nutznießern gehört der nationale und internationale Tourismus, der teilweise deutlich an die Erhaltung des Kulturerbes gebunden ist (McKercher/Du Cros 2002). Selbst in Gegenden des Massentourismus kann Kulturerbe dazu beitragen, die Touristenflüsse zu stabilisieren und zu diversifizieren, insbesondere außerhalb der Hauptsaison. Ein zweiter wichtiger Nutznießer ist die Immobilienwirtschaft. So übertreffen die Immobilienwerte von Wohnungen in gut erhaltenen Altstadtbezirken häufig diejenigen in Neubauvierteln. Auch Unternehmen, etwa forschungsintensive Firmen, konzentrieren sich gerne in kulturell reichen Gegenden historischer Städte (Europäische Kommission 2015).

Ähnlich vielschichtig, aber schwerer messbar ist der indirekte Nutzen von Kulturerbe, etwa durch Beiträge zur Lebensqualität der Menschen, zur Schönheit von Städten und Dörfern und zur Entwicklung einer örtlichen oder regionalen Identität. Sie gewinnen durch Prozesse wie Globalisierung, Digitalisierung und Migration weiter an Bedeutung (Tweed/ Sutherland 2007).

Entsprechend fordert der EU-Bericht, dass innovative Nutzungen von Kulturdenkmälern als Beitrag zu ökonomischem Wachstum und zur Schaffung von Arbeitsplätzen, zu sozialer Kohäsion und zur Nachhaltigkeit gefördert werden sollten. Dies soll sich auf alle drei Pfeiler der Nachhaltigkeit erstrecken: Ökonomie, Gesellschaft und Umwelt. In der ökonomischen Dimension geht es um die Förderung innovativer Finanzierungs-, Investitions-, Governance-, Management- und Geschäftsmodelle, um die Effektivität von Kulturerbe als ökonomischer Produktionsfaktor zu verbessern. In der sozialen Dimension geht 
es darum, durch Kulturerbe Integration, Inklusion, Kohäsion und Partizipation zu unterstützen. Für die ökologische Dimension wird angestrebt, durch Inwertsetzung von Kulturerbe zu einer nachhaltigen Entwicklung europäischer Kulturlandschaften beizutragen (Europäische Kommission 2015).

\section{Vorhergehende Diskurse im Naturschutz}

Ein ganz ähnlicher Paradigmenwechsel war bereits vor ca. 20 Jahren im Naturschutz zu beobachten. So waren auch die Anfänge des Naturschutzes stark von intrinsischen Motiven geprägt (»nature for itself«) (Mace 2014); mit zunehmender Umweltzerstörung gelangte die Philosophie, Natur vor den störenden Einflüssen des Menschen zu bewahren, an Bedeutung (»nature despite people«). Etwa um die Jahrtausendwende hingegen setzte sich dann ein ökonomischer Blick auf den Naturschutz durch. Als zentraler Ansatz der sogenannten »neuen Naturschutz-Wissenschaft" (Kareiva/Marvier 2012) setzte sich das Konzept der Ökosystemleistungen durch, das die vielfältigen Leistungen der Natur für den Menschen (»nature for people«) betont und auch ökonomisch bewertet. Kennzeichen dieser Ökosystemleistungen ist, dass sie erst allmählich von der Gesellschaft wahrgenommen, anerkannt und entgolten werden. Beispielsweise regulieren Großschutzgebiete das Klima, indem sie $\mathrm{CO}_{2}$ speichern. Sie stellen sauberes Trinkwasser bereit und sind insbesondere für naturnahen Tourismus und die Naherholung wichtig. Das Konzept der Ökosystemleistungen ist innerhalb der globalen Natur- und Umweltschutzpolitik in kurzer Zeit zum »Mainstream «Thema avanciert (Plieninger et al. 2013). So betont etwa die Biodiversitätsstrategie der EU (Europäische Kommission 2011): »Die Biodiversität [...] ist [...] unser Naturkapital, denn sie stellt Ökosystemdienstleistungen bereit, die die Grundlage unserer Wirtschaft bilden. Verschlechterung und Verlust dieses Kapitals stellen die Bereitstellung dieser Dienstleistungen in Frage und führen zum Verlust an Arten und Lebensräumen, des Wohlstandswertes der Natur und von Arbeitsplätzen und gefährden unser eigenes Wohlbefinden.«

Mittlerweile ist das Konzept der Ökosystemleistungen etwa in der Diskussion um das weltweite Schutzgebietsnetz fest verankert. So wurde in der 2010 veröffentlichten TEEBStudie (The Economics of Ecosystems and Biodiversity) der Wert der von den rund 120.000 Naturschutzgebieten der Welt erbrachten Ökosystemleistungen auf 4,4 bis 5,2 Mrd. USDollar pro Jahr geschätzt (TEEB 2011). Ein solches naturschutz-ökonomisches Denken wurde auch politisch aufgenommen, indem etwa die »Förderung des Nutzens von Biodiversität und Ökosystemleistungen für alle Menschen« im Strategischen Plan der UNÜbereinkunft zur biologischen Vielfalt für 2011-2020 als strategisches Ziel definiert wurde. In Ziel 11 wird spezifiziert, dass künftige Schutzgebietsausweisungen insbesondere Gebiete von besonderer Bedeutung im Hinblick auf Biodiversität und Ökosystemleistungen berücksichtigen sollen. Die Wiederherstellung und Förderung von Ökosystemleistungen ist auch im Lima Action Plan für das weltweite Netz der UNESCO-Biosphärenreservate als zentrales Ziel definiert (Plieninger et al. 2016). 
Was genau ist aber der Mehrwert des Ökosystemleistungs-Ansatzes für den Naturschutz? Im TEEB-Bericht (TEEB 2012) wird argumentiert, dass er insbesondere zum Aufbau breiter Allianzen für den Naturschutz beitragen kann, indem er eine Schärfung des gesellschaftlichen und politischen Bewusstseins für den Wert der Natur fördert. Aufgrund seiner engen Bezüge zu Aspekten des menschlichen Wohlbefindens kann er der Integration von Naturschutz in andere Politikfelder sowie in Fachdisziplinen jenseits von Naturschutz und Landschaftsplanung zum Durchbruch verhelfen, indem er die Implikationen von Biodiversität und Ökosystemen für Sektoren wie beispielsweise die Landwirtschafts-, Energie-, Klima-, Wasser-, Tourismus- und Gesundheitspolitik veranschaulicht sowie in die Raumordnung (Landes-/Regionalplanung) vermehrt einfließen lässt.

Darüber hinaus kann das Konzept zu einer verbesserten Akquise von Fördermitteln beitragen: Über den Ansatz können zusätzliche Mittel für das Schutzgebietsmanagement erschlossen werden, wenn Privatpersonen und Wirtschaftsunternehmen als tatsächliche Nutzer konkreter Ökosystemleistungen finanziell in deren Schutz und Bereitstellung eingebunden werden.

Schließlich kann der Ansatz Planungs- und Managemententscheidungen unterstützen, indem er ermöglicht, Konflikte zwischen verschiedenen Ansprüchen an Großschutzgebiete zu ermitteln. Auch macht er Leistungen sichtbar, die bislang noch kaum als solche betrachtet wurden (Plieninger et al. 2016).

Allerdings haben der Ökosystemleistungs-Ansatz und die mit ihm - wie Kritiker meinen - einhergehende »neoliberale Invasion« (Barlösius 2008) auch gewaltige Konflikte im Naturschutz ausgelöst. Schröter et al. (2014) verweisen auf insgesamt sieben solcher grundlegenden Debatten:

1. Der Ansatz wird als anthropozentrisch kritisiert. Andere hingegen argumentieren, dass er sich auch jenseits rein instrumenteller Werte bewegen kann.

2. Dem Ansatz wird vorgeworfen, durch sein Nutzen-Denken zur weiteren Ausbeutung der Natur durch den Menschen beizutragen. Andere stellen wiederum fest, dass er dazu beitragen kann, stärkere Bindungen zwischen Mensch und Natur herzustellen, indem er die menschliche Abhängigkeit von der Natur betont.

3. Es gibt Befürchtungen, dass der Ansatz zu Konflikten mit Zielen des Biodiversitätserhalts führt, während andere die Komplementarität dieser Ziele hervorheben.

4. Der Ansatz wird wegen seines Fokus auf ökonomische Bewertung in Frage gestellt. Andere hingegen argumentieren, dass sich die Ökosystemleistungen auf vielfältige, auch nicht-monetäre Werte beziehen.

5. Am Konzept wird die Förderung der Kommodifizierung der Natur kritisiert, während andere herausheben, dass die meisten Ökosystemleistungen nicht mit marktbasierten Instrumenten verknüpft sind.

6. Gängige Definitionen und Klassifikationen werden als vage kritisiert, während andere genau darin einen Vorteil für die transdisziplinäre Zusammenarbeit sehen. 
7. Das Konzept wird als normativ kritisiert, da es alle Wirkungen ökosystemischer Prozesse als wünschenswert beurteilt. Andere sehen in der normativen Natur des Ansatzes kein Problem, solange sie explizit gemacht wird.

\section{Chancen und Risiken einer Übertragung auf die Denkmalpflege}

Eine verstärkt ökonomische Inwertsetzung von Ökosystemen ist im Naturschutz ca. 15 Jahre früher erfolgt als in der Denkmalpflege, wo verblüffend ähnliche Debatten über eine »Kommodifizierung des Kulturerbes" geführt werden (Soini/Birkeland 2014). Welche Chancen hat eine Übertragung des Ökosystemleistungs-Ansatzes auf den Bereich des Kulturerbes? Ein Mainstreaming der Bedeutung von Kulturerbe in verschiedene Politikfelder fördert sicherlich eine zunehmende politische Bedeutung der Denkmalpflege - wobei dies politische Auseinandersetzungen um unterschiedliche Verständnisse von »Kulturerbe« zur Folge haben kann, wie etwa die Konflikte um die historische Rekonstruktion der Potsdamer Mitte zeigen. Auch kann die ökonomische Inwertsetzung zur Schärfung des gesellschaftlichen Bewusstseins von Kulturerbe beitragen und in stärkerem Maß als bisher private Mittel für den Denkmalschutz erschließen helfen. Allerdings sollten Lehren gezogen werden aus den Debatten um die »Ökosystemleistungen « und es sind teils schwierige Fragen zu beantworten. Wie sollten etwa unter einer neuen Sichtweise auf Kulturerbe Denkmäler bewahrt werden, denen kein direkter ökonomischer Nutzen gegenübersteht? Welche Auswirkungen hat eine »Ökonomisierung « auf gesellschaftlich umstrittene Arten von Kulturerbe, etwa aus der Zeit des Sozialismus? Wie geht man mit Zielkonflikten zwischen verschiedenen Kulturerbe-Werten um, beispielsweise aus verschiedenen Epochen, wie sie in Potsdam zwischen dem preußischen und dem sozialistischen Kulturerbe bestehen? Kann die Ökonomisierung des Kulturerbes zu einem Rückzug des Staats führen, etwa zu Einsparungen oder zu abgeschwächten ordnungsrechtlichen Regeln? Wie können Aspekte der sozialen Gerechtigkeit berücksichtigt werden?

\section{Literaturverzeichnis}

Ahmad, Yahaya (2006): The scope and definitions of heritage: From tangible to intangible. In: International Journal of Heritage Studies 12, 292-300.

Barlösius, Eva (2008): Die Eroberung natürlicher Prozesse und Phänomene - ein Beispiel für die »neoliberale Invasion «? Eine essayistische Annäherung. In: Die Gesellschaft der Unternehmen - Die Unternehmen der Gesellschaft. Gesellschaftstheoretische Zugänge zum Wirtschaftsgeschehen. Hg. von Andrea Maurer und Uwe Schimank. Wiesbaden: VS Verlag für Sozialwissenschaften, 252-261.

Europäische Kommission (2011): Our Life Insurance, our Natural Capital: an EU Biodiversity Strategy to 2020. http://eur-lex.europa.eu/legal-content/EN/TXT/?uri=CELEX:52011DC0244 (25.09.2018).

Europäische Kommission (2015): Getting Cultural Heritage to Work for Europe. Report of the Horizon 2020 Expert Group on Cultural Heritage. https://ec.europa.eu/programmes/horizon2020/en/news/gettingcultural-heritage-work-europe (25.09.2018). 
Europäische Kommission (2018): Strategie Europa 2020. https://ec.europa.eu/info/business-economy-euro/ economic-and-fiscal-policy-coordination/eu-economic-governance-monitoring-prevention-correction/ european-semester/framework/europe-2020-strategy_de (25.09.2018).

Harrison, Rodney (2015): Beyond »natural« and »cultural« heritage: Toward an ontological politics of heritage in the age of anthropocene. In: Heritage and Society 8, 24-42.

Kareiva, Peter; Marvier, Michelle (2012): What is conservation science? In: BioScience 62, 962-969.

Lowenthal, David (2005): Natural and cultural heritage. In: International Journal of Heritage Studies 11, 81-92.

Mace, Georgina M. (2014): Whose conservation? Changes in the perception and goals of nature conservation require a solid scientific basis. In: Science 345, 1558-1560.

McKercher, Bob; Du Cros, Hilary (2002): Cultural tourism: The partnership between tourism and cultural heritage management. Oxon: Routledge.

Plachter, Harald; Rössler, Mechtild (1995): Cultural landscapes: Reconnecting culture and nature. In: Cultural Landscapes of Universal Value: Components of a Global Strategy. Hg. von Bernd von Droste, Harald Plachter und Mechtild Rössler. Jena: Gustav Fischer Verlag, 15-18.

Plieninger, Tobias; Trommler, Kathrin; Bieling, Claudia; Gerdes, Holger; Ohnesorge, Bettina; Schaich, Harald; Schleyer, Christian; Wolff, Franziska (2013): Ökosystemleistungen und Naturschutz. In: Handbuch Naturschutz und Landschaftspflege. 28. Erg. Lfg. 04/13. Hg. von Werner Konold, Reinhard Böcker und Ulrich Hampicke. Weinheim: Wiley VCH Weinheim, 14 pp.

Plieninger, Tobias; Kizos, Thanasis; Bieling, Claudia; Dû-Blayo, Laurence L.; Budniok, Marie-Alice; Bürgi, Matthias; Crumley, Carole L.; Girod, Geneviève; Howard, Pip; Kolen, Jan; Kuemmerle, Tobias; Milcinski, Grega; Palang, Hannes; Trommler, Kathrin; Verburg, Peter H. (2015): Exploring ecosystem-change and society through a landscape lens: Recent progress in European landscape research. In: Ecology and Society 20.2. https://www.ecologyandsociety.org/vol20/iss2/art5/ (25.09.2018).

Plieninger, Tobias; Woltering, Manuel; Job, Hubert (2016): Implementierung des Ökosystemleistungs-Ansatzes in deutschen Biosphärenreservaten. In: Raumforschung und Raumordnung 74, 541-554.

Rössler, Mechtild (2006): World Heritage cultural landscapes: A UNESCO flagship programme 1992-2006. In: Landscape Research 31, 333-353.

Schröter, Matthias; van der Zanden, Emma H.; van Oudenhoven, Alexander P. E.; Remme, Roy P.; Serna-Chavez, Hector M.; de Groot, Rudolf S.; Opdam, Paul (2014): Ecosystem services as a contested concept: A synthesis of critique and counter-arguments. In: Conservation Letters 7, 514-523.

Soini; Katriina; Birkeland, Inger (2014): Exploring the scientific discourse on cultural sustainability. Geoforum 51, 213-223.

TEEB (2011): The Economics of Ecosystems and Biodiversity in National and International Policy Making. London: Earthscan.

TEEB (2012): The Economics of Ecosystems and Biodiversity in Local and Regional Policy and Management. London: Earthscan.

Throsby, David (2010): The Economics of Cultural Policy. Cambridge: Cambridge University Press.

Tweed, Christopher; Sutherland, Margaret (2007): Built cultural heritage and sustainable urban development. In: Landscape and Urban Planning 83, 62-69.

\section{Bildnachweis}

1 Europäische Kommission 2015. 